
Revue de Ihistoire des religions
Revue de l'histoire des religions
1 | 2019
Corps, ascèse et extinction dans l'histoire du bouddhisme (Inde, Corée, Japon)

\title{
Corrélations entre le corps et la doctrine bouddhique dans le shugendô d'Akyûbô Sokuden (Xvi siècle)
}

The correlation between the body and Buddhist doctrine in the shugendô of Akyûbô Sokuden (16 $6^{\text {th }}$ century)

Alexandre Goy

\section{OpenEdition}

Journals

Édition électronique

URL : https://journals.openedition.org/rhr/9406

DOI : $10.4000 /$ rhr.9406

ISSN : 2105-2573

Éditeur

Armand Colin

Édition imprimée

Date de publication : 1 mars 2019

Pagination : 99-120

ISBN : 978-2-200-93230-5

ISSN : 0035-1423

Référence électronique

Alexandre Goy, "Corrélations entre le corps et la doctrine bouddhique dans le shugendô d'Akyûbô Sokuden (xvie siècle) ", Revue de l'histoire des religions [En ligne], 1 | 2019, mis en ligne le 01 janvier 2022, consulté le 08 janvier 2022. URL : http://journals.openedition.org/rhr/9406 ; DOI : https:// doi.org/10.4000/rhr.9406 


\section{Corrélations entre le corps et la doctrine bouddhique dans le shugendô d'Akyûbô Sokuden ( $\mathrm{XVI}^{\mathrm{e}}$ siècle)}

La présente contribution propose de mettre en avant le travail du moine Akyûbô Sokuden, un des premiers intellectuels japonais à avoir tenté au $X V I^{e}$ siècle de définir la voie de l'ascèse religieuse dite shugendô, dont la tradition a longtemps été tenue secrète, et de présenter la place et la fonction que tient le corps du pratiquant au cour de cette voie ésotérique dans son ceuvre majeure, le Shugen shûyô hiketsu-shû ou Recueil des arcanes essentiels à la pratique du shugen (1521-1528). Les mises en corrélations entre le corps du shugenja ou du yamabushi, les accessoires, les pratiques posturales et la doctrine bouddhique issue du mahāyāna montrent comment l'auteur rattache cette tradition ascétique à la recherche du surgissement de la bouddhéité présente en chaque être.

The correlation between the body and Buddhist doctrine in the shugendô of Akyûbô Sokuden (16 ${ }^{\text {th }}$ century)

This paper seeks to highlight the work of the monk Akyûbô Sokuden, one of the first Japanese scholars to have tried, in the $16^{\text {th }}$ century, to define the ascetic religious practice of shugendô, whose tradition was kept secret for many years. The paper also describes the place and function of the adept's body, at the heart of this esoteric path, in his major work, the Shugen shûyô hiketsu-shû (1521-1528). The correlations he draws between the body of the shugenja or of the yamabushi, his accessories and postural practices and Buddhist doctrine resulting from the mahāyāna show how the author links this ascetic tradition to the desire to bring out the Buddhist present in every human being. 


\section{INTRODUCTION $^{\mathrm{I}}$}

Le shugendô («voie du shugen », ou « voie de réalisation (gen) dans l'exercice $(s h u)$ [d'ascèses] $)^{2}$ est un sujet encore peu connu de la recherche occidentale malgré l'intérêt qu'il ne manque jamais de susciter chez les spécialistes de la question religieuse, des historiens, des ethnologues ou des spécialistes de la civilisation japonaise ${ }^{3}$. Le shugendô est un mouvement religieux complexe ${ }^{4}$ qui, au cours de son histoire, a puisé ses références dans le taoïsme, la voie du yin et du yang, le bouddhisme ésotérique, et les différentes formes du culte shinto. Il s'appuie sur un panthéon partagé entre les grandes figures du bouddhisme, et les divinités locales ${ }^{5}$. On trouve trace de sa pratique à l'écrit dès le $\mathrm{X}^{\mathrm{e}}$ siècle au Japon ${ }^{6}$. Celle-ci est toujours active de nos jours ${ }^{7}$ malgré une discontinuité de ce mouvement à partir du XVI ${ }^{\mathrm{e}}$ siècle $^{8}$.

1. Afin d'harmoniser les références bibliographiques, nous avons opté pour mettre le prénom suivi du patronyme de l'auteur y compris pour les auteurs japonais.

2. On doit la première tentative de définition de ce mouvement au spécialiste du bouddhisme Enkû Uno (1885-1950) dans un article du troisième volume de l'imposant recueil Nihonshûkyô daikôza (Traité sur les religions du Japon), Tôkyô, Toho Shohin, 1929.

3. On notera l'apport de Gaston Renondeau et Carmen Blacker, les travaux d'Hartmut Rotermund, Byron Earhart, Anne Bouchy, Allan Grapard, Heather Blair, principaux spécialistes du shugendô en Occident.

4. La recherche occidentale, principalement anglo-saxonne, présente régulièrement ce mouvement comme «syncrétique». Depuis les années 1980, la recherche japonaise lui préfère le terme de «composite » ou «complexe » (shûgô shûkyô) qui est directement issu de l'histoire des sciences des religions au Japon. Voir Fumio Tamamuro, Minkan shinkô chôsa seiri handobukku (Guide de références pour l'analyse des croyances populaires), Tôkyô, Yuzankaku, 1987. L'expression se réfère également aux doctrines « d'harmonisation », de " conciliation » développées à l'époque de la fusion shinto bouddhique (shinbutsu shûgô) entre le VIII et le XIV ${ }^{\mathrm{e}}$ siècle.

5. Il faut rappeler que les figures bouddhiques et shintos ont toujours été l'objet de cultes combinés très peu de temps après l'introduction du bouddhisme au Japon au $\mathrm{VI}^{\mathrm{e}}$ siècle. L'école du Hossô-shû notamment, le moine Gyôki (668-749) en tête, fut la première à exposer les doctrines composites de ce qui deviendra le ryôbu-shintô (la voie des divinités des deux mandalas), c'est-à-dire la manifestation des figures bouddhiques à travers les divinités shintos.

6. Première mention du shugen dans le Nihon sandai jitsuroku, Chroniques des trois ères du Japon (969 ap. J.-C.).

7. Les lieux de pratique les plus dynamiques aujourd'hui sont principalement les trois centres religieux de Kumano, le Mont Kôya, la chaîne montagneuse d'Ômine et la région de Yoshino, ou encore la région de Nikkô.

8. Suivi d'une totale interdiction due à «loi de prohibition de la pratique shugen 》 (shugen kinshirei), promulguée en 1872 et visant à lutter contre les superstitions. Cette interdiction fut levée le 3 mai 1947 avec l'inscription de la liberté de culte dans la Constitution japonaise. 
Pour comprendre les origines, floues, du shugendô, il faut s'intéresser à l'histoire des localités où se trouvent les grands centres religieux sis au plus proche de l'environnement naturel ${ }^{9}$. Notamment les régions de Kinpusen et Ômine (département de Nara) ou Kumano (département de Wakayama). Ces lieux profitèrent de la poussée des nouvelles écoles du bouddhisme ésotérique issues de Chine, le Tendai et le Shingon au VIII ${ }^{\mathrm{e}}$ siècle. Ces nouvelles formations établirent un nombre conséquent de temples et monastères en montagne, mais aussi de plus petites structures. Par exemple, des halls de réclusion (dôsha) utilisés pour accueillir les processions d'été et les cérémonies d'offrandes de fleurs, ou de petits sanctuaires, montés à la faveur des pérégrinations des moines durant les périodes de purification et d'oblation $\left(\operatorname{tos} \hat{O}^{10}\right)$.

9. Jusqu'à l'ordonnance sur les grands travaux de 1889 et l'accélération de l'exploitation agricole, les forêts représentaient les $2 / 3$ du territoire occupé à $71 \%$ de zones montagneuses. Un large culte des montagnes (sangaku shinkô) nourri de croyances populaires, participait déjà des activités religieuses au Japon au $\mathrm{VIII}^{\mathrm{e}}$ siècle (observation de la littérature confirmée par des fouilles archéologiques menées dès 1983 dans les régions centrales du Honshû). L'environnement spirituel était alors figuré par l'expression «autre-monde» (takai, ce terme pouvant se référer aux berges des cours d'eau, au "pays » au-delà des mers, aux zones d'ombre à l'abri du regard, aux sommets des montagnes) et le culte des montagnes relevait d'éléments identifiés par la recherche japonaise comme suit : les pratiques du culte pouvaient être liées aux divinités dispensatrices de l'eau (mikumari no kami), aux divinités patronnes des communautés rurales (shugojin, devenues plus tard protectrices du territoire du japon et de la loi bouddhique), aux représentants

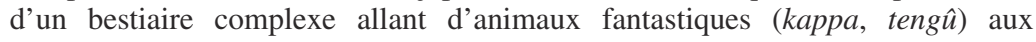
esprits frappeurs ou courroucés (niki mitama, ara mitama). La montagne était également observée comme le lieu de transit des esprits des défunts, son culte pouvant impliquer la présence de maîtres de la possession ou de médiateurs tels que les officiants de sanctuaires shintos locaux (kannushi), les figures érémitiques (hijiri) ou les ritualistes itinérants. Voir Kunio Yanagita, Yanagita Kunio zenshû (Yanagita Kunio, auvres complètes), Tôkyô, Chikuma shobô, 1997, ou Tsutomu Tokieda, Shugendô no kôkogaku kenkyû (Études archéologiques sur le Shugendô), Tôkyô, Yûzankaku, 2005.

10. Ce mouvement de décentralisation obligea la mise en place d'une série de réglementations: "Ceux des moines et nonnes qui, s'engageant dans la pratique méditative, recherchent l'apaisement de l'esprit et souhaitent s'éloigner des souillures pour pratiquer le jeûne en montagne, doivent avoir l'accord des Superviseurs du Conseil. [...] Il nous faut toujours connaître la montagne de résidence [du pratiquant]. Les déplacements ne seront pas tolérés ». Extrait de l'article «Réglementation des moines et nonnes » (Sôniryô), 701, Codes de l'ère Taihô (Taihô ritsuryô), à lire dans Shigeru Gorai, Shugendô no shugyô to shûkyôminzoku (La pratique du Shugendô dans le folklore religieux), Gorai Shigeru chosakushû 5, Kyôto, Hôzôkan, 2008, p. 13. 
Après de multiples interdictions ${ }^{11}$, la pratique d'ascèse en extérieur (sanrin shugyô) fut officiellement reconnue en $812^{12}$ avec la nomination d'un administrateur officiel de la région de Kumano (kumano bettô), le moine Kaikei, fils d'un ministre de la cour impériale. Le recours à la pratique expérimentale ${ }^{13}$ en complément de l'étude théorique favorisa alors l'établissement de nouveaux centres religieux dans des montagnes plus éloignées de la capitale, créant une activité cénobitique et encourageant un culte direct des buddhas, bodhisattvas et esprits protecteurs de la famille impériale, dépassant le cadre des activités ritualistes tenues par le shinto et le bouddhisme d'état, centrés autour de l'empereur.

La géographie du Japon ne peut être considérée comme une géographie de l'inconnu. C'est une géographie habitée, entretenue par une importante mythologie ${ }^{14}$. Aussi, de grandes figures d'autorité comme les empereurs retirés ${ }^{15}$ finirent de donner à ces lieux toute leur puissance symbolique et participèrent à y transférer définitivement l'administration religieuse. À l'exemple de l'empereur Shirakawa (1053-1129), qui parcourut en pèlerinage la chaîne montagneuse d'Ômine dix fois, l'empereur Toba (11031156), qui répéta ce même exercice douze fois, l'empereur Goshirakawa (1127-1192), trente-quatre fois, et l'empereur Gotoba

11. Après la réglementation de 701 , la pratique en extérieur fut interdite en 717 et 729 . Le prêche et la lecture de sūtras en extérieur interdits en 764 . Un ascète itinérant (sanrin shugyôja), le moine Dôkyô (?-772), fut évincé de la secte Sanron en 770, jusqu'alors sous la protection de l'état aristocratique de l'empereur Shôtoku. Une interdiction de résider à l'extérieur du temple fut établie en 799 en conséquence de l'interdiction de tenir une estrade d'ordination non-officielle, d'établir un monastère sauvage, de diffuser la parole du Buddha en dehors des canaux de diffusion officiel dès 785 .

12. D'après le Kii zokufudoki (XIX siècle) donné dans Hitoshi Miyake, «Kumano bettô keizu no shakaiteki imi» («La signification sociale de la généalogie des administrateurs de Kumano »), in Tetsugaku 12, 1990, p. 363.

13. Aux précédents exemples peuvent être ajoutés les exercices de concentration (zenjô), récitations de sūtras et offrandes, invocations (nenbutsu) et prières aux divinités (kajikitô), purifications (arai) et visites de lieux sacrés (shuhai), entre autres.

14. Dont les éléments les plus connus ont été transmis à travers le Kojiki et le Nihonshoki (712 et 720), ou encore les recueils de légendes (fudoki) composés à partir de 713. Voir à ce propos Alain Rocher, Mythe et souveraineté au Japon, Paris, PUF, 1997 ; ou François Mace, « Le Kojiki, une Énéide longtemps oubliée ? », in Ebisu 49, 2013, p. 117-132.

15. Appellation qui désigne les empereurs qui continuèrent d'assurer la gouvernance, dans l'ombre de l'empereur en titre, depuis leur retraite monastique. 
(1180-1239), vingt-huit fois. En 1090, l'empereur Shirakawa se rendit également à Kumano ${ }^{16}$ et participa à la systématisation des activités en montagne en désignant lui-même l'administrateur des trois plus grands sanctuaires de la région. Cette période correspond aussi à la fixation de l'identité des différents sommets, la distribution spatiale du culte des divinités bouddhiques et assimilées (divinités kami) avec la réalisation de transmissions légendaires comme le Shozan engi (XII siècle, Antécédents des montagnes) ou le Kinpusen hon'engi (1133, Antécédents à l'origine de Kinpusen). C'est dans ce cadre que des organisations liées à la pratique d'une discipline ascétique se développèrent.

Dans le shugendô, le terme «ascèse » (shugyô), littéralement "pratique exercée », doit s'entendre comme une pratique travaillée assidûment, une pratique qui entraîne à la constance, qui détourne de l'échec. Une askèsis cathartique qui dépouille le corps et la pensée de ses impuretés, l'exercice d'un art, d'une discipline qui permet de se doter progressivement de la vérité. Le « shugendô» ne désignait alors qu'une méthode pour atteindre cette vérité (shugyô-hô) et non une structure organisée à la manière des grandes écoles bouddhiques ${ }^{17}$. Les pratiquants de cette voie étaient appelés «les gens du shugen» (shugenja) ou «ceux qui demeurent en montagne» (yamabushi). Qu'ils fussent moines ou laïques, l'objectif revendiqué était de s'enfoncer dans les forêts profondes ${ }^{18}$, de gravir les montagnes, de parcourir les cavernes et les cours d'eau afin de faire l'expérience directe du contact des divinités.

«Dans nos sommets se déploie la vertueuse quintessence de l'existence foncière spontanée, la Terre pure du double plan de la Matrice et du Diamant. Les montagnes majestueuses [forment] l'estrade complète des neuf assemblées du Diamant. Les cavernes éparpillées sont les réceptacles du Lotus à huit pétales de la Matrice.

16. Voir à ce propos Arnaud Brotons, «Lecture ethnologique d'un lieu saint connu depuis le VIII ${ }^{\mathrm{e}}$ siècle », in Ateliers no 30, 2006, p. 139-165.

17. Les premières assemblées de pratiquants du shugen apparurent officiellement au XII ${ }^{\mathrm{e}}$ siècle dans le Shugen tokudo sahô, Processus d'intégration du shugen du moine Jôken (1161-1231), même si, d'un point de vue légendaire, le Shozan engi ( $\mathrm{XII}^{\mathrm{e}}$ siècle) décrit un premier rassemblement au $\mathrm{VII}^{\mathrm{e}}$ siècle de plus de 380 pratiquants du shugen autour du personnage d'En no Gyôja, qui deviendra bien plus tardivement le saint patriarche des tenants du shugendô.

18. L'entrée en montagne (mine'iri ou nyûbu) est le premier pas du pratiquant sur la réalisation de sa voie. 
Les montagnes, les rivières, les plantes et les arbres sont l'incarnation directe de Shana (Vairocana). Le vent sur les sommets, l'écho des vallées incarnent l'enseignement du Corps de la Loi. Les vénérés des trois fractions s'y alignent en multitude. [...] De fait, le visible et l'audible originels y sont perceptibles. [...] Ce sont les montagnes des forces agissantes où l'on s'unit aux trois subtilités, dans ce vertueux milieu dont la nature véritable restait jusqu'alors inconnue. $»^{19}$

La mobilité des pratiquants les amenait à composer avec un treillis socio-culturel très vaste et participait à l'ouverture de nouveaux lieux de cultes sur des sites appelés «montagnes habitées/de forces agissantes »(reizan). Ces lieux étaient divisés en aires d'exercice ( $d \hat{o} j \hat{o})$ et les activités ascétiques y étaient organisées selon la nature des divinités présentes et l'efficacité de leur culte. L'évolutivité de ce mouvement est donc particulièrement importante, et ses variations étroitement liées à l'histoire locale.

La première et unique tentative d'agencement de ces multiples organisations apparaîtra aux alentours du XVII siècle avec la consolidation de deux grands groupes de pratiquants, le Tôzan-ha et le Honzan-ha ${ }^{20}$. Rappelons ici la notion de «voie » (dô) qui est très importante dans le shugendô, car elle définit ce mouvement non pas comme une institution religieuse homogène ${ }^{21}$ qui se baserait sur l'étude et l'engagement cénobitique, mais bien comme un «chemin » que l'on pratique de façon libre et volontaire. La transmission de ses préceptes est longtemps restée orale et

19. Shugen-sĥu, troisième rouleau, «En 1 : À propos de remarques à tenir sur la lustration rituelle. ». Toutes les traductions proposées ici, à moins d'un renvoi à leur auteur, sont personnelles. En raison des limitations liées à la forme de l'article, les analyses et explications nécessaires à la compréhension d'un essai doctrinal tel que celui de Sokuden sont réduites à leur strict minimum. Ces précisions et la traduction complète du Shugen shûyô hiketsu-shû sont accessibles dans ma thèse doctorale soutenue à l'Université de Strasbourg en 2016 sous le titre Le Shugendô d'Akyûbô Sokuden dans le Japon médiéval. Écrire et transmettre une identité (à paraître).

20. En 1613, le gouvernement militaire de Tokugawa Ieyasu émit une « réglementation du shugendô » (shugendô hatto) qui imposa l'assimilation des différentes confréries par le Tôzan-ha (d'affiliation Shingon) ou le Honzan-ha (d'affiliation Tendai). Si cette loi permit la reconnaissance officielle du shugendô, elle marqua aussi son déclin. La vie nomade et les ermitages furent interdits, les pratiquants durent se fixer dans les monastères ou les communautés villageoises. L'ordination fut obligatoire, les pratiques réglementées et limitées par l'autorité. L'enseignement de la voie fut étouffé par le corps doctrinaire du bouddhisme ésotérique officiel.

21. Une école de pensée ou une tradition, nommées «sĥे » comme dans les termes « religion» $($ shûkyô $)$ et « tradition zen » (zensh $\hat{u})$. 
secrète, avant l'arrivée de théoriciens de la voie entre le XIV et le $\mathrm{XV}^{\mathrm{e}}$ siècle. Il en découle une place importante de l'agir, à travers la méditation, la récitation ou la gestuelle, sans distinction de l'origine des pratiquants. Ainsi, le mouvement shugendô ne se réclamait d'aucun dogme, mais finit de codifier ses gestes sous l'effort de personnalités religieuses charismatiques, au premier rang desquels le moine Sokuden.

\section{Akyûbo Sokuden et Le ShugEN-SHÛ}

On trouve très peu d'informations sur le personnage de Sokuden $\mathrm{Akyûbô}^{22}$, si ce n'est celles qu'il fournit lui-même. Auteur de cinq essais doctrinaux composés en kanbun (lettres classiques en chinois) conçus sur la forme de recueils de feuillets ${ }^{23}$, ce moine membre de la communauté shugen de $\mathrm{Nikkô}^{24}$, formé au bouddhisme Tendai, pratiquant des monts Kinpusen puis figure influente de la montagne Hikosann ${ }^{25}$, a participé à la formalisation de sa voie en combinant et déterminant des éléments jusque-là épars de la pratique shugen. Au cours d'un long périple à travers le $\mathrm{Japon}^{26}$, Sokuden a fait un lourd travail de sélection dans l'optique

22. «Akyûbô », littéralement « la demeure du souffle de la lettre A ».

23. Feuillets volants, kirigami. Notes, transmissions, réflexions souvent cryptiques qui accompagnent la pratique. Elles se limitent la plupart du temps à l'énumération de gestes ou de schémas. Ces papiers furent transmis dès l'époque Kamakura (1185-1333) entre les générations de pratiquants. Non destinés à l'archivage, ils furent cependant à l'origine des premiers recueils de pratiques.

24. D'après Sokuden lui-même dans le seizième feuillet du Hikosan shugen hiketsu injinshû, Recueil de témoignages secrets du shugen de la montagne Hiko (1521-1558). Première édition connue en 1920.

25. Département de Fukuoka.

26. Initié au shugen dans la montagne Nikkô-yama avant 1504 , il traversa le pays en direction de l'Ouest et obtint son premier titre de guide conducteur de la voie du shugen au pavillon Renzô-in en 1524 dans la région de Hiko, sous l'autorité du moine Shô'un. En 1525, il reprit la route en direction du Nord, s'arrêta pour pratiquer dans une grotte près du temple Natadera, puis dans les hauteurs des montagnes de Hakusan (département d'Ishikawa). Il continua quelque temps son voyage avant de rentrer dans la région de Hiko en 1544. Il reprit alors sa formation au Reisenji auprès du moine Akô avant de commencer à enseigner à son tour à travers tout le pays entre 1550 et 1558 . D'après les informations transmises par Sokuden et ses disciples dans le Sanbu sôchô hôsoku mikki, Notes secrètes des lois et traditions des trois sommets (à partir de 1525), première édition connue en 1709, et dans le Hikosan shugen hiketsu injinshû. 
de créer une identité au shugendô et de lui donner les moyens discursifs d'échanges, de transmission et d'influence avant d'être finalement choisi pour intégrer les auteurs canoniques du shugendô contemporain $^{27}$.

La première copie connue du Shugen-shû date de 1691. Elle est complétée d'une note d'introduction donnée par le moine Fue ${ }^{28}$. Titré «Introduction aux feuillets du shugendô » («Shugendô kirigami hatsudai »), cet addendum identifie Sokuden comme le compilateur officiel de l'ouvrage, bien que son nom ne figure pas sur le document original. Cependant, Sokuden signa ses autres recueils entre 1521 et 1558 et l'on trouve à l'article «À propos des stèles [dressées] en montagne » du Shugen-shû la date de «la troisième année de l'ère Daiei » (1523), qui n'est pas explicitement donnée comme date de composition, mais qui correspond bien à la période d'activité de Sokuden.

Il existe une version xylographique de cette compilation datée de 1692, dans les collections du musée Guimet ${ }^{29}$. L'ouvrage présente la même note d'introduction que la version de Fue, mais précise que l'impression du document serait due à un certain Nakano Rokuemon. L'objet est trop abîmé pour servir de support de traduction. Imprimé sur une seule feuille pliée, le livret est relié par des coutures sur un seul côté dans le plus pur style fukurotoji. Un sous-titre a été apporté à cette version : « Renkakuden sokuden kaisen » (« Transmission du moine Renkaku révisée par Sokuden »). C'est la version la plus ancienne qu'il m'ait été donné de voir et l'examen visuel m'a permis d'identifier une complète similarité avec l'édition contemporaine.

Cette dernière édition fut pour la première fois imprimée en juillet 1798 par le Hannya-in, temple Shingon de la province de Satsuma (actuelle préfecture de Kagoshima). Un des membres de

27. Les textes en question sont le Shugen shûyô hiketsu shû, le Shugen tongaku sokushô shû, Recueil de faits qui amènent à une compréhension rapide du shugen (1521-1569), tous deux de Sokuden, le Sanjûsan tsûki (Les trente-deux feuillets du shugen) des moines Renkaku et Chikô, le Shugen shinanshô (Abrégé du shugen), auteur inconnu, et l'En no kimi gyôshôki (Récit des différentes vies d'En no kimi/En no gyôja). Voir Tetsuya Sasaki, in Hitoshi Miyake (dir.), et Nihon Daizôkyô Hensankai, Shugendô shôso kaidai (Commentaires au Shugendô shôso), Tôkyô, Kokusho kankokai, 2000.

28. Ryômei Fukui, in Hitoshi Miyake, ibidem, p. 233. shû.

29. Qui conserve également une version de 1693 du Shugen tongaku sokushô 
cette communauté, le moine Junshin, en signe la préface. C'est cette version qui se retrouve à l'origine de ma traduction, et que l'on peut lire dans la compilation Shugendô shôso de Tatsue Nakano, elle-même incluse dans la collection Nihon Daizôkyô (Collection des canons bouddhiques du Japon, 48 volumes) ${ }^{30}$.

Les plus anciens recueils de pratiques shugen connus sont le Yamabushi-chô, Notes des yamabushi - deux rouleaux dont un seul nous est parvenu-(probablement 1366), le Buchî kanjô honki, Règles fondamentales pour le rituel de lustration en montagne (XIV siècle), l'Ozasa hiyôroku, Enregistrements secrets d'Ozasa (XIV $V^{\mathrm{e}}$ siècle), et l'Aozasa hiyôroku, Enregistrements secrets d'Aozasa $\left(\mathrm{XV}^{\mathrm{e}} \text { siècle }\right)^{31}$. Ces ouvrages se composent de peu de documents (deux à six feuillets). Il ne s'agit pas là d'essais théoriques mais d'énumérations, parfois commentées, de pratiques en extérieur. Ces textes présentent des énoncés moins fournis et structurés que l'ouvrage de Sokuden, peu de définitions, mais apportent de précieuses informations sur la richesse des activités en montagne. Ces feuillets sont souvent trop concis pour pouvoir certifier qu'ils participent de l'ouvrage de Sokuden. Mais leur diffusion semble attester de leur importance dans la tradition orale du shugendô.

D'après Sokuden lui-même ${ }^{32}$, le matériau d'origine de son travail lui aurait été fourni durant l'ère Eishô (1504-1521) par son maître, le moine Shô'un, sous la forme de cinquante feuillets d'enseignements ${ }^{33}$. C'est trois feuillets de plus que le Shugen-shu, mais il demeure impossible aujourd'hui de savoir ce qu'il en est advenu. De plus, l'introduction du Shugen-shû de 1691 insiste, lui, sur l'apport des moines Chikô et Renkaku. Dans le Shugen tongaku

30. Le Shugendô shôso fut réédité, entre 1973 et 1978, par la compagnie Suzuku gakujutsu zaidan, puis, en 2000, par la société Kokusho kankôkai avec un addenda dirigé par le professeur Hitoshi Miyake (Shugendô shôso kaidai, Commentaires au Shugendô shôso). Cette compilation se présente en trois volumes. Le premier, édité d'abord en 1917, rassemble un grand nombre de textes ayant trait au Tôzan-ha. Le deuxième et le troisième volume (première édition en 1920) se répartissent les données consacrées au Honzan-ha et les centres religieux indépendants comme celui du mont Hikosan, ainsi que des récits historiques et fictionnels de la fondation des lieux de pratique. Le Shugen shû appartient au deuxième volume de cette compilation.

31. Hitoshi Miyake, Shugendô girei no kenkyû (Étude des rites du Shugendô), Tôkyô, Shunjusha, 1999 (Édition augmentée), p. 799.

32. En notes du Hikosan shugen hiketsu injin shû, Recueil de témoignages des secrets du shugen de Hikosan (1558).

33. À la fois par écrit et sous forme de transmissions orales. 
sokushôsh $\hat{u}$, Sokuden fait précisément état de la réception de trentetrois feuillets, dont on peut dire qu'il s'agit du recueil Sanjûsan tsûki composé par le moine Renkaku, abbé du temple Hikosan reizanji, et le moine Chikô, guide conducteur de la voie dans le même établissement. Ces derniers auraient eux-mêmes obtenu ces notes du haut-patriarche En no gyôja, ancêtre légendaire des yamabushi.

L'étude de ce dernier recueil permet effectivement de noter de nombreuses similarités et donc de confirmer cette transmission, même si celle-ci a dû avoir lieu indirectement (Renkaku aurait vécu aux alentours du XII ${ }^{\mathrm{e}}$ siècle ${ }^{34}$, et Chikô se tint deux générations avant celle de Sokuden). Si le travail de Sokuden est effectivement une reprise du Sanjûsan tsûki, il s'agit d'une version plus complète : elle présente quarante-sept feuillets, là où la première version n'en propose que trente-trois. Sokuden y fait aussi plus volontiers référence aux canons de la littérature bouddhique japonaise et aux sūtras transmis au Japon par des échanges avec la Chine.

Le Shugen shû se divise en trois parties et classe les propos de Sokuden en quarante-sept feuillets. La première partie comprend des vues sur la mise du pratiquant (itai, douze feuillets) et les premières clés de compréhension de la pratique (sept feuillets). La deuxième traite de détails ésotériques (sept feuillets) et de concepts attachés aux différents rituels (sept feuillets). Dans la dernière partie sont réunis des us et coutumes plus généraux (shiyô, sept feuillets), la présentation des accréditations, les rapports de pratiques, les lignées des pratiquants, et une notice (soesho, sept feuillets).

\section{EXPÉRIENCE DU CORPS ET ASCÈSE D'EXPOSITION}

Dans sa compilation, Sokuden s'adresse principalement aux pratiquants déjà largement engagés dans la voie du shugendô. Son contenu, bien que difficile à déchiffrer, est détaillé et référencé, et sa forme agencée et didactique. Dans le shugendô proposé par Sokuden, l'ascèse est une pratique centrale qui permet de dépasser le carcan du monde physique en sublimant le corps. Le détachement par la voie de transcendance n'est nullement un abandon doloriste

34. Ryômei Fukui, in Hitoshi Miyake Shugendô shôso kaidai..., p. 235. 
du corps, comme en ont malheureusement donné l'exemple des pratiquants du shugendô à l'origine plus contemporaine ${ }^{35}$.

$«[\ldots]$ En ne rejetant pas ce corps obtenu sur le plan des kamis [on atteint] avec facilité le stade de la grande vacuité. C'est là le secret que porte ce corps. $»^{36}$

Il ne s'agit pas de «nier le monde, être hostile à la vie, mépriser les sens et se passer d'eux $»^{37}$, mais au contraire de voir le corps comme un véhicule, un outil de transmission.

«Si on se conforme au cadre de l'écrit, devenir Buddha [semble] un avenir très lointain. Il est nécessaire de faire simplement l'expérience du corps de la Loi de Diamant qui existe foncièrement en ce corps, en s'éveillant sur sa nature propre de Buddha. Ce n'est qu'après que l'on [peut] s'intéresser aux enseignements des différentes écoles. $»^{38}$

Ainsi, expérience et incarnation sont les maîtres mots des théories d'ascèses présentées par Sokuden. Le corps du pratiquant incarne l'unité. Il est issu de la plus basse condition de l'être, et cependant capable de lui conférer le statut d'un éveillé (buddha) dans le temps présent.

«Le yamabushi. Il est l'incarnation [du principe d']être Buddha en ce corps. Il se tient au stade ultime, celui du souverain des éveillés Biru (Vairocana). $»^{39}$

« Dans le Sütra du Lotus, il est dit que la nature de Vairocana est la pureté. [Mais] incarnés dans les trois domaines et les cinq mauvaises destinées, nous sommes tous également soumis aux illusions et sombrons dans [la répétition du] cycle de vie et de mort. Par le biais du savoir de vérité, nous faisons l'expérience de la révélation suprême. $»^{40}$

«D'après les canons, on enseigne que les caractéristiques réelles de la chair et de l'esprit de l'ensemble des êtres seront toujours [issus] du corps de sapience de Vairocana. Il faut prendre cela en considération. C'est véritablement là le cœur de la pratique d'entrée en montagne. C'est l'enseignement direct de la plus haute idée du shugen. Comment

35. Voir à ce propos l'histoire du yamabushi Jitsukaga (Anne Bouchy, « Jitsukaga, yamabushi des premières années de Meiji, et le shugendô », in Revue de l'histoire des religions 193/2, 1978, p. 187-211), ou celle de Tetsumonkai (Andô Kösei, « Des momies au Japon et de leur culte », in L'homme, 8/2, 1968, p. 5-18.

36. Shugen-shû, premier rouleau, «En 12 : À propos des jambières ».

37. Friedrich Nietzsche, Généalogie de la morale, in Gérard Mendel (éd.), Euvres philosophiques complètes, vol. 7, 1971, p. 305.

38. Shugen-shû, premier rouleau, «En 1: Â propos de l'adoption ou du rejet de textes de références ».

39. Ibid. «En 1 : À propos de la calotte».

40. Ibid. «En $6:$ À propos du discernement entre vice et vertu». 
notre important précepte [ne] se pourrait-il être "devenir Buddha en ce corps ?" ${ }^{41}$

L'exercice de l'ascèse entrepris à travers ce corps amène le pratiquant à se détacher de tout ce qui ne saurait être utile à son exhaussement en lui apprenant à sublimer le bon (l'élevé) et à abandonner le mauvais (le commun). Pour cela, il doit prendre possession et afficher les constituants sensibles du bon. Cette pratique doit ainsi lui permettre de prendre conscience, de faire l'expérience concrète (intime) de ce qu'il y a de bon en lui.

Sokuden va ainsi exposer ce qu'il revendique être propre au shugendô, en hiérarchisant ses données en trois classes: «l'abordable » (senryaku), « le profond » (shinpi), «le cryptique » (kyokuhi). Cette classification impose au lecteur d'assimiler les éléments qui composent la voie par différents paliers d'appréhension, du plus manifeste au plus implicite, afin d'obtenir les clés pour accomplir sa pratique ascétique jusqu'à atteindre le stade d'un buddha (sokushin jôbutsu).

L'ouvrage s'ouvre sur un descriptif précis, en douze feuillets, de la silhouette du pratiquant et des archétypes qui lui sont attribués. Aujourd'hui, ce descriptif fait toujours référence ${ }^{42}$ et a permis aux formations modernes de continuer de faire valoir l'uniforme du yamabushi.

De fait, le corps disparaît, car il n'est plus que la réunion d'attributs symbolisés dans un ensemble d'accessoires. Il s'agit pour Sokuden de la forme idéalisée du yamabushi. Le pratiquant et les outils qui l'accompagnent sont désignés sous le terme « enveloppe » (itai), c'est-à-dire la formalisation sensible des principes de la voie. La description de Sokuden suit une logique verticale qui reprend les étapes de la naissance : la tête (chapeau et calot), le torse (surtout, habit, accessoires à main et coffres), puis les jambes (tablier et chausses).

Le lecteur est ainsi directement introduit à la pensée shugen par l'intermédiaire du corps du pratiquant. Pour Sokuden, comme pour

41. Ibid. Deuxième rouleau, «En 1 : À propos de la pratique des Dix Plans ».

42. On peut, par exemple, le retrouver dans les préceptes modernes enseignés par Iyano Biho, guide conducteur du shugen de Nikkô. Voir Biho Iyano, Me kara uroko no shugendô : daishizen no naka no shinbutsu ga toku uchû no chie to satori eno michi (Un aperçu du Shugendô : la voie vers la sapience et l'éveil dans un univers décrit par les divinités de la nature), Tôkyô, Gakken, 2006. 
la majorité des tenants du bouddhisme japonais, le monde sensible est régi par la forme. Elle provoque la discrimination, mais elle est aussi universelle. Et même si celle-ci n'est jamais désignée comme étant « véritable », elle représente pour Sokuden le seul moyen de communication, d'exposition de la Loi.

« À la question, pourquoi dans le shugendô doit-on absolument porter de tels accessoires et veiller ainsi à refuser les différences? La réponse est : la mise des pratiquants qui commence par le calot et se termine par les jambières tout au long de douze [accessoires] permet de percevoir le corps de la Loi, le Buddha à travers ce corps. Ainsi, en revendiquant ces accessoires, on expose le sens profond des attributs que nous partageons. $»^{43}$

De ce fait, la forme doit être parfaite et suivre une codification complexe à même de transmettre le bon message. Par exemple, il est aisé d'assimiler symboliquement un couvre-chef à la notion de «protection». Mais lorsque ledit couvre-chef « $[\ldots]$ a pour caractéristique une forme circulaire, il incarne [alors] l'accomplissement des cinq stades. [C'est pourquoi on lui donne une] circonférence de cinq shaku $(\sim 151 \mathrm{~cm})$ et un diamètre d'un shaku, six sun et 7/10 [de sun] $(\sim 51 \mathrm{~cm}) »$, devenant à même de créer du sens et communiquer, aux initiés, les bases d'une pensée profonde.

Dans le Shugen-shû seront ainsi bien souvent mis en corrélation éléments concrets et éléments spéculatifs. Sokuden semble vouloir s'assurer que le pratiquant formalise son savoir au travers de références aisément représentables et donc, plus simples à transmettre. L'identification, c'est-à-dire le modèle, est dominante dans le travail de Sokuden afin d'amener l'arrière-plan conceptuel à transcender la forme :

«Selon les transmissions écrites: dans yamabushi, le caractère "yama", la montagne, se compose de trois traits verticaux et d'un trait horizontal 山. Les trois traits verticaux correspondent aux trois corps : de la Loi, de rétribution, et d'émanation ; et les trois apodicticités [de l'incarnation] : de la vacuité, de l'impermanence, de l'équilibre. Le trait horizontal correspond [au principe] de l'harmonie des trois corps et de l'immédiateté des trois apodicticités. Dans "bushi", allongé/ caché, le caractère est constitué de l'association du signe "homme" et “chien" 伏. Le caractère "homme" rend compte de la nature absolue de l'être et le caractère "chien" rend compte de l'obscurité. Obscurité et

43. Shugen-shû, troisième rouleau, «En 5: À propos du dialogue en cinq articles ». 
nature absolue ne font qu'un en dépit de leur dualité [car il ne faut pas confondre] le proche et l'éloigné [soit le principe] de dénominations différentes dans une incarnation unique. $\gg^{44}$

Pour Sokuden, la particularité du shugendô provient donc de la possibilité qu'il offre aux pratiquants de proposer une exposition, une exemplification directe des principes de la Loi bouddhique. Une communication physique entre les hommes et leur environnement spirituel sans discrimination.

«À la question: la voie du shugen est-elle identique à l'exotérisme? La réponse est: elle n'est ni exotérique ni ésotérique. Elle est un procédé qui tranche avec les dispositifs philosophiques complexes [pour trouver] l'unité des deux enseignements. Éloignée [du principe] d'un enseignement basé sur les [trois] écrins elle exprime directement l'essentiel de l'esprit du Buddha. C'est ce qu'on appelle "la transmission intime d'un esprit à un autre". Après avoir fait l'expérience de la pratique, on atteint sans attendre les conditions mentales propres à la non-incarnation. On parle là de "la connaissance expérimentale de l'éveil individuel." ${ }^{45}$

\section{SPATIALISATION DU CORPS ET ENTRÉE DANS LA MONTAGNE}

Pour Sokuden, le pratiquant n'est donc pas un être «transformé ». Il reste tel quel et c'est sous cette forme qu'il atteint l'Éveil.

«Il ne s'agit pas d'être rétribué du statut de Buddha en résultat d'un accomplissement. L'idée [revendiquée par le] shugen est que nous sommes des maṇdala dont le matériau originel est le plan de la Matrice et le plan du Diamant qui correspondent à notre chair et à notre esprit. C'est donc sans modification du pratiquant en lui-même que sera expérimentée l'expression éveillée de Shana (Vairocana). La chair, c'est le mandiala de la Matrice dont l'origine provient des cinq éléments. L'esprit, c'est le maṇdala du Diamant, dont l'origine provient de l'élément de la conscience. ${ }^{46}$

Les exercices d'ascèse ont donc pour but de lui faire prendre conscience, par expérimentation, de l'excellence de sa nature. Que l'on soit « ordinaire » ou «élevé », quelle que soit l'enveloppe qui nous a été rétribuée, l'expérimentation du Buddha, son «intime réalisation », en ce corps est possible. Sokuden engage donc les

44. Ibid. Deuxième rouleau, «En 1 : À propos des deux caractères de "yamabushi" ».

45. Ibid. Premier rouleau, «En $4:$ À propos des trois dialogues ».

46. Ibid. Deuxième rouleau, «En 1 : À propos de la pratique des Dix Plans ». 
yamabushi à faire « retour» à la vérité de leur être et à éveiller leur conscience grâce aux preuves que transmet leur corps dans la pratique. Cela passe aussi par l'appropriation du cadre de l'ascèse, les montagnes, et le franchissement de différents stades, figurés par la «traversée des dix plans », et personnifiés dans la figure du Roi de sapience, Fudô myôo (Acalanâtha) ${ }^{47}$.

L'entrée en montagne relevait, en règle générale, d'un exercice processionnaire. Elle pouvait durer près d'une centaine de jours. Des groupes de yamabushi, encadrés d'une équipe de supérieurs de différents grades, les guides conducteurs de la voie (sendatsu), pénétraient le flanc des chaînes montagneuses en suivant un itinéraire soigneusement sélectionné et éprouvé par les pratiquants les plus expérimentés. De nombreux gîtes, sanctuaires ou temples, attendaient de recevoir la procession et marquaient une étape dans la progression physique et mentale du yamabushi. Chacune de ces étapes était l'occasion de procéder à une ascèse soit immédiate, soit engagée sur la durée. Le corps, centre d'attention du rite, traversait ainsi symboliquement l'ensemble des plans d'incarnation possibles afin de s'ouvrir au plan des buddhas.

«La voie tire son sens de la traversée de l'ensemble des Dix plans. La pratique de l'entrée en montagne est naturellement fonction des caractéristiques de chacun des Dix plans, exactement comme un chemin [s'adapte aux courbes de la montagne]. [Ainsi] quand est produit l'esprit d'éveil, il s'agit de l'éveil parfait de la lettre vaṃ, [issu de] la lettre A. $»^{48}$

« Nos sommets sont les maṇdala dont l'existence foncière se trouve être le plan de la Matrice et celui du Diamant. Ce sont des endroits reculés [mais] connectés aux Dix plans. C'est là qu'ont lieu les deux entrées en montagne qui sont dites "régulière" et "inversée". L'entrée régulière en montagne, c'est le déroulement logique [qui procède] des coproductions conditionnées issues de l'obscurité. Il s'agit de l'exercice dit "partant de la cause aboutir à la conséquence". [Ou] Prendre origine sur le plan des Enfers et aboutir au plan des buddhas. La pratique en montagne inversée, c'est l'expression des coproductions conditionnées issues de [la connaissance] de la nature absolue. Il s'agit de l'exercice "partant de la conséquence se tourner vers la cause". [Ou] Prendre origine sur le plan des buddhas et aboutir au plan des Enfers. Les Dix

47. Dans la tradition bouddhique, celui-ci apparaît d'abord comme un serviteur de Mahāvairocana (jp. Dainichi). Il est représenté sous une forme courroucée (funnu) dont la fonction, entre autres, est d'asservir les ennemis de la Loi.

48. Ibid. «En 3 : À propos des quatre titres ». 
plans sont composés des six [espaces] des êtres ordinaires et des quatre [espaces] des êtres supérieurs. ${ }^{49}$

«L'exercice des quatre [espaces] des êtres supérieurs [est une pratique reposant sur] l'examen du principe. Dans l'exercice des six [espaces] des êtres ordinaires, la première des voies [à emprunter] est celle des Enfers. [La pesée des] actes $^{50}$. La deuxième des voies est celle des esprits faméliques. [L'interdit des] céréales ${ }^{51}$. La troisième des voies est celle des bêtes. [L'interdit de] l'eau ${ }^{52}$. La quatrième est celle des guerriers éternels. [La pratique de] la lutte ${ }^{53}$. La cinquième est celle des Hommes. [La pratique du] repentir ${ }^{54}$. La sixième est celle des divinités. [La pratique de] la danse de longévité ${ }^{55}$. Il s'agit là de rituels pour les novices, [leur permettant d'obtenir] des forces extérieures les moyens adaptés selon [le principe de] la grande compassion qui amène [les buddhas à] se substituer aux Hommes dans la souffrance. [...] Venons-en à l'exercice des quatre [espaces] des êtres supérieurs qui correspond aux plans des auditeurs, des éveilléspour-soi, des bodhisattvas et des buddhas. [Celui qui] a prêté attention à l'enseignement des quatre [nobles] vérités et a pris conscience de la relation de causes à conséquences, on dit de lui que c'est un auditeur. [Celui qui] a perçu les douze coproductions conditionnées et a pris conscience du cycle de vie et de mort, on dit de lui que c'est un éveillé-pour-soi. [Celui qui] a fait vœux d'une grande compassion et a pratiqué les six perfections, on dit de lui que c'est un bodhisattva. [Celui qui], être complet, a atteint l'Éveil individuellement et a conduit les autres à l'Éveil, on dit de lui que c'est un buddha. [...] Ceci étant, les pratiques des quatre [espaces] des êtres supérieurs telles que présentées dans le shugen ne sont pas du tout celles-ci. [Nos] pratiques

49. Ibid. «En 1 : À propos de la pratique des Dix Plans».

50. Le contenu de ces gestes n'est jamais explicité par Sokuden. Gôbyô. Il s'agit d'un véritable exercice de pesée où le pratiquant est attaché à un contrepoids (la Pierre de l'Immuable, fudô ishi). S'il est plus lourd que le contrepoids, c'est que ses péchés sont intolérables.

51. Kokudachi. Ayant rejeté toute nourriture céréalière (principalement le blé, l'avoine, le riz, le millet et le seigle), le pratiquant commence à se nourrir exclusivement des fruits de la forêt, mokujiki. Il peut s'agir d'écorces, de racines, de glands. Cette pratique dure traditionnellement de sept à quatorze jours.

52. Suidan. Interdiction de boire ou de se laver pendant une période déterminée.

53. Sumô. Une véritable opposition physique entre deux pratiquants. Cette pratique était très courante durant les cérémonies shintos d'apaisement des esprits, et s'appuie en partie sur la légende, présentée par le Kojiki, de la réclusion de la divinité Amaterasu, pour qui on organisa des luttes afin de l'attirer à l'extérieur de sa grotte.

54. Zange. Le pratiquant se présente devant son supérieur et demande miséricorde pour ses actes mauvais et ses pensées illusoires.

55. Ennen mai. Cette pratique, développée durant l'époque Heian, était devenue très populaire à l'époque médiévale. Comme son nom l'indique, il s'agissait de réclamer par cette danse la protection de divinités dans l'espoir d'atteindre un âge canonique. 
se basent uniquement sur l'examen du principe, la réalité instantanée. Autrement dit, la réalité de la souffrance qui coïncide avec le corps de la Loi. La réalité de l'origine de la souffrance qui coïncide avec la révélation suprême. La réalité de la cessation [de la souffrance] qui coïncide avec le nirvāṇa et la réalité de la voie qui coïncide avec la nature individuelle [de chacun]. Telle est [pour nous] la pratique des auditeurs. Les passions qui coïncident avec la révélation suprême, l'entrave des actes qui coïncide avec [la vertu] d'élucidation, la voie de la souffrance qui coïncide avec l'état de quiétude. Telle est [pour nous] la pratique de l'éveillé-pour-soi. [La pratique] des six perfections sansattributs, tel est [pour nous] l'exercice du bodhisattva. $»^{56}$

« Le yamabushi est l'action personnifiée de Fudô [qui s'exprime] en son corps. [Il a donc pour lui] l'intime réalisation de la consubstantialité des Dix plans. Ainsi, l'étole nouée [autour du pratiquant] représente pleinement la logique subtile de l'identité unique des Dix plans, que détient en l'essence le pratiquant dans son corps, et de l'harmonie des trois corps du Buddha. D'après les documents secrets, l'étole nouée est l'habit du corps de la Loi, elle rentre dans la catégorie des trois vêtements religieux. Cette étole possède neuf bandes. Considérant que neuf bandes font neuf liens, les neuf nœuds sont les neuf plans [liés entre eux]. Tous les êtres des neuf plans sont liés dans ces neuf bandes. Le pratiquant se vêt de cet habit. Les neuf bandes sont les neuf plans, et le pratiquant incarne lui le plan des buddhas. Ainsi, la nomme-t-on aussi le "vêtement des Dix plans inséparables". Le Roi de Sapience Fudô est l'incarnation de la complétude des Dix plans. Il est au stade le plus élevé de l'union du commun et de l'élevé. Les Dix plans ne sont qu'une seule réalité, il n'y a donc pas de distinction entre les êtres vivants et les buddhas. L'égarement et l'illumination forment une seule incarnation, la compréhension et l'incompréhension se confondent. C'est véritablement l'expression de la fusion des Dix plans. C'est un espace unique où se réalise une prise de conscience. $»^{57}$ «Ainsi, l'esprit du pratiquant, quel qu'il soit, peut accueillir le Roi de Sapience Fudô. Le réceptacle intime du noble lotus est de nature spirituelle et individuelle. Fudô de la Grande Lumière porte en lui le Buddha primordial [Dainichi]. $»^{58}$

«Autrement dit, les flammes qui nimbent le corps [de Fudô représentent] les Enfers. Son allure noire [représente le plan des] esprits faméliques. L'oiseau de feu Garuḍa [représente le plan des] bêtes. L'épée effilée de sa main droite [représente le plan des] guerriers éternels. La cordelette [représente] l'agrégation des formes humaines. Les bijoux sur son corps [représentent les] attributs subtils des divinités [qui président au Ciel]. Son vêtement, [représente les] deux véhicules des auditeurs et des éveillés-pour-soi. Le chapelet de pierres précieuses de sa main gauche [représente le] cordon de Grande compassion des

56. Ibid.

57. Ibid. Premier rouleau, «En 4 : À propos de l'étole nouée ».

58. Ibid. Deuxième rouleau, «En 6: Â propos des Dix plans du Roi de Sapience Fudô ». 
bodhisattvas. Le lotus sur son front [représente] le plan des buddhas. Voilà ce qu'il en est dit dans le Sūtra du Vénéré Immuable : "Ce Grand Roi de Sapience ne demeure pas ici. Mais voilà, il réside à l'intérieur de chacun des êtres." $\gg^{59}$

Avant l'entrée en montagne, le pratiquant est accueilli dans un environnement consacré. C'est là qu'il reçoit un premier enseignement et qu'on lui délivre la vérité cachée en son corps. Les premières formations de yamabushi se réunissaient déjà dans des gîtes de montagne ou des bâtiments entretenus par les monastères bouddhiques, les nagatoko ${ }^{60}$. Ces bâtisses, lieux de prière pour les fervents, devaient accueillir les pratiquants shugen avant le début des processions afin qu'ils reçoivent les premiers rituels de purification (goma) et les cérémonies d'initiation assises (tokogatame). La répartition des places était alors codifiée selon le niveau d'expérience du pratiquant et son rôle durant les pérégrinations de groupe (en charge de l'eau, du bois, de l'encadrement des rites...). Le corps du pratiquant y était associé aux cinq éléments tels qu'ils se retrouvent, selon le bouddhisme ésotérique, dans l'ensemble des phénomènes de ce monde et repris par Sokuden comme suit :

« D'après l'enseignement [de nos maîtres]: lors de l'entrée en montagne régulière, on [se positionne en] abaissant la main droite et en levant la main gauche. Lors de l'entrée en montagne inversée, on abaisse la main gauche et on lève la main droite. La position en demi-tailleur s'applique [indifféremment des deux]. Le pied est placé à un shaku et deux sun du siège $(37 \mathrm{~cm})$. Lors de l'entrée en montagne d'été, qui correspond à la direction entre bois majeur (Est, Nord-Est) et bois mineur (Est, Sud-Est), durant le cycle de Jupiter, il faut, de gauche comme de droite, de haut en bas, prendre conscience [de son corps]. Se rapporter à la tradition orale. $»^{61}$

"Pour ce qui concerne le Renfort de l'assise au gîte de montagne. "Toko", "le fond" (l'assise), ce sont les mandala des deux sommets du Diamant et de la Matrice. C'est le lieu de pratique où coexistent le commun et l'élevé. "Katame", "le solide", ce sont les cinq éléments inébranlables du corps de la Loi. Ensemble ils donnent sa forme rituelle

59. Ibid.

60. Ou raidô, espace de transition entre la cour intérieure d'un temple et le bâtiment où sont généralement exposés les trésors. On peut retrouver une illustration de l'usage du nagatoko dans le Chôshuki, Journal d'un long automne (1130) de Minamoto no Morotoki. Hitoshi Miyake, Shugendô to nihonshûkyô (Le Shugendô et les religions du Japon), Tôkyô, Shunjûsha, 1996, p. 52.

61. Shugen-shû, deuxième rouleau, «En 2: Â propos du Renfort de l'assise [pratiqué] en montagne ». 
au principe d'être Buddha en ce corps. Notre plus haut patriarche Dainichi l'ainsi-venu [...] est l'incarnation de la parfaite roue des multiples vertus qui est sans début ni fin. Dans cette incarnation sont compris les cinq éléments de la terre, de l'eau, du feu, du vent et de l'éther. [...] Ces cinq éléments sont sans limites, ils se répandent à travers les Dix plans parmi les êtres pensants et non pensants. ${ }^{62}$

Dans un autre feuillet, Sokuden propose une illustration visuelle de l'organisation du lieu où étaient accueillis les groupes de pratiquants avant l'entrée dans la montagne. L'orientation du dessin rappelle celui des diagrammes géométriques des maṇualas avec l'Est figuré vers le haut, l'Ouest au bas, le Nord à gauche et le Sud à droite. Les yamabushi étaient répartis de part et d'autre d'un brasier en position assise pour incarner les cinq étages d'un stûpa. Situer ainsi le corps dans l'espace c'est donner du sens à la présence du pratiquant dans cet environnement. Sokuden continue ainsi à créer une connexion entre les phénomènes sensibles de ce monde, qu'il s'agisse du corps du yamabushi, du lieu de pratique, des montagnes environnantes, toujours pour simuler l'unité essentielle de la forme.

« Tout être capable de faire preuve de patience peut devenir Buddha en ce corps et, assis comme il se doit, appréhender la forme véritable [de toutes choses]. ${ }^{63}$

«Au préalable, [on prend connaissance de] sa place pour le Renfort de l'assise. Bois majeur (Est Nord-Est) pour le plan du Diamant, bois mineur (Est Sud-Est) pour le plan de la Matrice. Ensuite, on produit le sceau des mains jointes autour du vide horizontal. Le sceau étant formé à l'horizontale, les cinq éléments sont à la verticale. [On récite le] chant de la lettre germe "vam". Puis, on produit le sceau des mains jointes autour du vide à la verticale. Le sceau étant formé à la verticale, les cinq éléments seront à l'horizontale. [On récite le] chant de la lettre "vaṃ". Ensuite, on s'allonge sur le flanc. Il faut plier le coude droit et réaliser l'élément du vent. On doit étendre le bras gauche, assurer [la position de ses] hanches et ajuster son assise. Il ne s'agit rien de moins que [de personnifier la lettre] VAN en position horizontale. Pour le Renfort de l'assise, c'est la lettre VAN en position verticale. Verticalité et horizontalité ne font qu'un. On trouve là le stade ultime des quatre postures. Il faut veiller à bien considérer le stūpa de la lettre "vạ̣" de la sphère de la Loi aux six éléments. » 


\section{LE CORPS ET SA CONSÉCRATION : LE RITUEL DE LUSTRATION (KANJÔ)}

Au moment d'entrer en montagne, un rituel de lustration est réalisé sur les pratiquants pour symboliquement les baigner dans la connaissance. Il était également répété à la suite de séries d'ascèse entreprises par le pratiquant afin de conclure son effort et de le ramener au monde des vivants, c'est-à-dire à l'espace présent, gratifié des efforts accomplis. La réalisation de ce rituel était suivie de la remise d'un talisman, assurant la renommée du pratiquant et donnant preuve de sa pérégrination. Encore un objet marquant le corps du yamabushi et lui permettant de revendiquer son expérience.

«En ce qui concerne le rituel de lustration à quatre degrés. Il s'agit, premièrement, du rituel de lustration d'entrée au gîte. Deuxièmement le rituel de lustration de la pesée des actes. Troisièmement, du rituel de lustration qui fait suite au jeûne de céréales. Quatrièmement, du rituel de lustration de la venue au monde. On nomme ces rituels de lustration à quatre degrés les rituels de l'Immuable. $»^{64}$

«"L'eau de consécration" est une expression indienne qui signifie "immaculé". "Sui", "l'eau", est sa transcription chinoise. Ainsi, l'eau est l'ingrédient vertueux de la pureté dans sa nature foncière, la source originelle de la double loi de rétribution du support et de l'être. De fait, tous les buddhas conçoivent leur nature grâce à l'eau, et l'ensemble des êtres conçoit sa matérialité grâce à l'eau. Selon, en troisième lieu, les transmissions écrites : le principe ainsi que l'activité des cinq connaissances sont présents dans l'eau. Premièrement, grâce à l'apaisante purification, qui est le propre de l'eau, on expose le corps matériel. C'est la connaissance du grand et parfait miroir. Deuxièmement, on fait se réfléchir dans le point central de l'eau l'ensemble des phénomènes. Non pas en profondeur, non plus en surface. C'est la connaissance d'égalité. Troisièmement, on fait se réfléchir dans le point central de l'eau l'ensemble des attributs tangibles, pour rendre compte des claires divergences. C'est la connaissance de l'appréhension sublime (le discernement). Quatrièmement, on amène l'ensemble des phénomènes à maturité grâce à l'eau. C'est la connaissance du devoir à accomplir. Cinquièmement, on ne retient pas cette eau. C'est la connaissance de l'incarnation de la sphère de la Loi. $\gg^{65}$

«Verser une eau foncièrement pure sur les yamabushi qui entrent pour la première fois en montagne, c'est révéler les cinq buddhas

64. Ibid. Troisième rouleau, «En 1: À propos de remarques à tenir sur la lustration rituelle ».

65. Ibid. Deuxième rouleau, «En 4: À propos de l'eau de la consécration [pratiquée] en montagne». 
inhérents à tous les êtres. [...] Avec l'eau de sapience des précédents buddhas, on asperge, "kan", le sinciput, "jô", des futurs buddhas. Il faut bien garder ceci à l'esprit. $»^{66}$

«À propos du talisman de l'eau de consécration. Selon les chroniques secrètes : le talisman de l'eau de consécration représente le stūpa du dhāraṇi qui renferme un trésor. Il s'agit [d'un certificat] de filiation [qui fait suite] à la lustration de l'entrée en montagne, le stūpa de l'unique lettre vam qui nous habite. Il est fabriqué à base de [planches de] saule. Sa longueur est d'un sun et huit bu $(5,5 \mathrm{~cm})$ qui représentent les dix-huit domaines du pratiquant. [La largeur] des quatre côtés associés est de trois sun et six bu $(11 \mathrm{~cm})$. Ce qui représente les six éléments interconnectés [dans les trois aires]. Les quatre lettres hūṃ, trāh, hrīḥ, ạ̣ sont respectivement notées sur les quatre faces. En dessous, doit figurer la lettre vam. Il s'agit en fait de représenter l'eau de la Loi des cinq connaissances. ${ }^{67}$

«L'eau de consécration représente la source originelle de la naissance. Le brasier rituel représente la mort. Les codes de l'entrée en montagne font pour cela référence à l'intra-utérin, le couvrechef, à l'extra-utérin, la Véritable lustration, à l'accouchement, l'eau de consécration, à l'anéantissement, le brasier. Il faut veiller à bien considérer ceci. $»^{68}$

"Même s'il est difficile de s'élever [au niveau] de Buddha, la lumière de la connaissance [se diffuse] bel et bien entre les êtres vivants et les buddhas. Avec nos maigres et futiles connaissances, nous n'avons pas le droit de douter de l'origine profonde de la nature véritable de toutes choses. Les huit pétales de notre part originelle s'épanouiront naturellement. La lettre A qui se tient à l'intérieur de nos cœurs nous apparaîtra distinctement dans sa juste vérité. Cette voie, que l'on arpente sans encombre, que l'on expérimente sans encombre, c'est cela la pratique de l'entrée en montagne. Il s'agit véritablement de l'exercice sublime de l'union en un cœur indivisible, l'expérience intime du lien étroit entre le relatif et l'absolu. » ${ }^{69}$

Arrivé au bout de son accomplissement, le pratiquant yamabushi est invité à répéter l'opération afin de faire montre de sa réalisation personnelle et d'être à même, par son expérience, d'apporter son soutien à d'autres pour les faire progresser sur la voie. Car l'objectif revendiqué par Sokuden dans la détermination d'une telle discipline n'est rien moins que d'arriver à élever individuellement le corps et l'esprit afin de faire usage de ses connaissances pour ouvrir la voie à ceux qui se tiennent encore dans «l'obscurité ».

66. Ibid.

67. Ibid.

68. Ibid. «En 6 : À propos du petit bois [ramassé] en montagne ».

69. Ibid. Troisième rouleau, «En 1: À propos de remarques à tenir sur la lustration rituelle». 
Le corps du pratiquant, sublimé par son expérience intime de la voie, couvert des attributs du bon, en contact permanent avec les figures religieuses les plus hautes, en pleine maitrise des environnements habités des figures les plus impressionnantes de la mythologie japonaise, devient le principal vecteur de diffusion du shugendô jusqu'à nourrir à son tour des légendes populaires.

«Il est dit dans les transmissions écrites: cette pratique de l'entrée en montagne, c'est l'exécution parfaite des six perfections du bodhisattva. C'est la règle [selon laquelle] une grande compassion [sera obtenue] pour les souffrances endurées. C'est ainsi désormais que chaque pratiquant prend avec lui son sistre de la grande compassion salvatrice, et sauve les êtres en portant leurs souffrances à travers les six perfections. C'est alors qu'il fait entendre le son du sistre [grâce auquel] les hommes voient les obstacles [à leur progression] s'évanouir par l'élimination des méfaits des trois activités. Et rapidement, ceux-ci se révèlent à l'état de Buddha. $»^{70}$

70. Ibid. Premier rouleau, «En 7 : À propos du sistre ». 